\title{
CONTAMINANTES ATMOSFÉRICOS Y SU VIGILANCIA
}

\section{Emiliano Aránguez (1), José María Ordóñez (1), Javier Serrano (2), Nuria Aragonés (1), Rosalía Fernández-Patier (3), Ana Gandarillas (1) e Iñaki Galán (1)}

(1) Consejería de Sanidad y Servicios Sociales. Comunidad de Madrid.

(2) Consejería de Medio Ambiente, Junta de Andalucía.

(3) Centro Nacional de Sanidad Ambiental. Instituto de Salud Carlos III.

\section{RESUMEN}

Se presentan algunos conceptos básicos relativos a la contaminación atmosférica. Aunque, desde un punto de vista sanitario, nuestro interés se centra en los efectos que producen los contaminantes sobre la salud de la población, es importante conocer cuáles son los principales contaminantes, sus fuentes emisoras, sus caracteristicas físicoquímicas, los métodos de captación y análisis que utilizan las estaciones de control de la contaminación atmosférica, los límites establecidos por la legislación vigente y las recomendaciones de la Organización Mundial de la Salud respecto a los niveles de inmisión.

Este trabajo repasa estos conceptos en relación con los contaminantes que se han analizado en el Estudio Multicéntrico Español de Contaminación Atmosférica y Mortalidad (EMECAM): partículas, dióxido de azufre $\left(\mathrm{SO}_{2}\right)$, dióxido de nitrógeno $\left(\mathrm{NO}_{2}\right)$, monóxido de carbono $(\mathrm{CO})$ y ozono $\left(\mathrm{O}_{3}\right)$. Para ello se han utilizado las publicaciones más recientes en la materia, incluyendo parte de lo que va a ser en un futuro inmediato el eje de actuación frente a la contaminación atmosférica: el nuevo conjunto de directivas de la Unión Europea (algunas en fase de propuesta) y las recomendaciones últimas (aún sin publicar) de la Organización Mundial de la Salud.

Por último, se plantea el amplio abanico de aspectos que incumben a la Salud Pública en el campo de la contaminación atmosférica, a pesar de que la vigilancia y control dependen administrativamente de las autoridades ambientales.

Palabras clave: Contaminación atmosférica. Salud pública. Partículas. Dióxido de azufre. Dióxido de nitrógeno. Monóxido de carbono. Ozono.

\section{ABSTRACT \\ Air Pollutants and the Monitoring Thereof}

Some basic concepts regarding air pollution are set out Although, from a health care standpoint, our interest revolves around the impact which pollution has on human health, it being important to ascertain the main pollutants, the sources of emissions, the physicochemical properties thereof, the sampling and analysis methods which are used at the air pollution control stations, the limits set by the laws currently in impact and the World Health Organization recommendations with regard to the levels of inmission.

This study reviews these concepts with regard to the pollutants which have been analyzed in the Spanish Multicenter Study of Air Pollution and Mortality (EMECAM): particles, sulfur dioxide $\left(\mathrm{SO}_{2}\right)$, nitrogen dioxide $\left(\mathrm{NO}_{2}\right)$, carbon monoxide $(\mathrm{CO})$ and ozone $\left(\mathrm{O}_{3}\right)$. For this purpose, the most recent publications on this subject have been used, including part of what is going to be the line around which all of the measures aimed at combating air pollution are going to be revolving in the very near future, that is, the new set of European Union Directives (some currently in the proposal stage) and the latest recommendations (not as yet published) of the World Health Organization.

Lastly, a wide range of aspects are set out which involve Public Health in the field of air pollution, despite the monitoring and control thereof falling to the environmental affairs autborities in terms of government organization.

Key words: Air pollution. Public health. Particles. Sulfur dioxide. Nitrogen dioxide. Carbon monoxide. Ozone.

Correspondencia:

Emiliano Aránguez Ruiz.

Servicio de Sanidad Ambiental y Antropozoonosis.

Consejeria de Sanidad y Servicios Sociales.

O'Donnell 52, piso $3 .^{\circ}$

28009 Madrid. 
«Me parece que vi a un grajo azul volando esta mañana. Pero el smog era tan denso que resultó ser un cardenal aguantando la respiración.»

Michael J. Cohen

\section{EL PROBLEMA DE LA CONTAMINACIÓN ATMOSFÉRICA}

Se entiende por contaminación atmosférica «la presencia en el aire de materias o formas de energía que impliquen riesgo, daño o molestia grave para las personas y bienes de cualquier naturaleza»' ${ }^{1}$. Siempre ha existido contaminación atmosférica de origen natural, por erupciones volcánicas, incendios, tormentas de arena, descomposición de la materia orgánica o polen, pero es a partir del descubrimiento del fuego por el hombre cuando aparece la contaminación atmosférica antropogénica o contaminación atmosférica en sentido estricto ${ }^{2}$, la cual ha cobrado importancia, sobre todo, a partir de la revolución industrial y el uso masivo de combustibles fósiles como fuente de energía ${ }^{3}$.

Aunque se puede originar contaminación atmosférica por escapes o arrastres de gases u otro material volátil, almacenado o utilizado de diversas formas, la principal fuente de contaminación son los procesos que implican combustión, al producirse la oxidación de los distintos elementos que componen los combustibles, las materias primas y el aire.

Así pues, los contaminantes pueden clasificarse desde su propia génesis. El carbono presente en los combustibles y el nitrógeno del aire, al oxidarse en los proccsos dc combustión, generan dióxido de carbono (gas no tóxico pero con importantes efectos indirectos sobre el medio ambiente y la salud, por su contribución al recalentamiento global) y óxidos de nitrógeno respectivamente. Otros elementos constituyentes de los combustibles y materias primas determinan la emisión de partículas, óxidos de azufre, compuestos orgánicos volátiles (COV), dioxinas, furanos, etc. Por su parte, procesos de combustión incompletos generan la emisión de monóxido de carbono, partículas y bifenilos policlorados, entre otros.

Las fuentes de emisión de contaminantes atmosféricos de origen antropogénico pueden ser puntuales, generalmente fijas y de gran caudal de emisión, como es el caso de las grandes factorías aisladas de otras instalaciones industriales, o pueden ser zonales, es decir, una mezcla de fuentes fijas y móviles de diferente entidad y agrupadas en el espacio, donde vienen a coincidir también con la población que sufre los efectos de la contaminación ${ }^{4}$.

Sin embargo, el conocimiento de los contaminantes y de las fuentes de emisión no abarca el complejo proceso de la contaminación atmosférica. Las características estructurales y dinámicas de la atmósfera y las características morfológicas del terreno determinan la dispersión de los contaminantes en el espacio y su evolución temporal. La dispersión configura la diferente concentración de contaminantes en la atmósfera (inmisión) en la zona de influencia de la fuente emisora. La evolución de los contaminantes conlleva la aparición de otros nuevos no emitidos por la fuente directamente: son los contaminantes secundarios, como el ozono formado a partir de los óxidos de nitrógeno y los compuestos orgánicos volátiles en presencia de la luz solar, o el ácido sulfúrico formado a partir de la oxidación del dióxido de azufre.

Aunque la determinación de la exposición individual depende de múltiples factores (ritmos de actividad, relación entre exposición en ambientes exteriores y ambientes interiores, laborales y no laborales), se puede afirmar que el conocimiento de los valores de inmisión en el aire ambiente nos acerca al de la dosis a la que está expuesta la población posibilitando el estudio de sus efectos en salud. Este hecho ha justificado históricamente el control y la vigilancia de los niveles de inmisión de contaminación atmosférica. 


\section{PRINCIPALES CONTAMINANTES ATMOSFÉRICOS}

El dióxido de azufre y las partículas derivadas de la oxidación de combustibles fósiles son los principales contaminantes atmosféricos urbanos del mundo y, por ello, los más estudiados, tanto dentro como fuera de España. El presente artículo contempla, además del dióxido de azufre $\left(\mathrm{SO}_{2}\right)$ y las partículas, otros contaminantes de interés: el monóxido de carbono $(\mathrm{CO})$, el dióxido de nitrógeno $\left(\mathrm{NO}_{2}\right)$ y el ozono $\left(\mathrm{O}_{3}\right)$. Para todos ellos, las fuentes de emisión antropogénicas y concentraciones de fondo conocidas aparecen en la tabla 1.

Las redes de vigilancia de la contaminación atmosférica miden valores de inmisión, es decir, la concentración en el aire de cada contaminante. Las medidas más usadas para expresar esta concentración son $\operatorname{los} \mathrm{mg} / \mathrm{m}^{3} \mathrm{o}$ los $\mu \mathrm{g} / \mathrm{m}^{3}$, aunque también se usan las partes por millón (ppm), por lo que se presentan las equivalencias en la tabla 2.

Las medidas de los contaminantes en el aire, aunque se realicen de forma continua, se expresan en promedios, dependiendo de los objetivos que se planteen. En relación con la exposición crónica, se utilizan promedios anuales, de 24 horas, o de ocho horas para los contaminantes con mayor oscilación a lo largo del día $\left(\mathrm{CO}_{\text {y }} \mathrm{O}_{3}\right)$. Los promedios diarios, pese a haber sido los más utilizados, ya sólo se aplicarán en la futura normativa europea a las partículas y al $\mathrm{SO}_{2}$. En relación con las exposiciones agudas se utilizan los valores máximos horarios o, incluso, de 30,15 o 10 minutos.

A continuación se comentan brevemente algunas características de los principales contaminantes atmosféricos.

\section{Partículas en suspensión}

El término «partículas en suspensión» abarca un amplio espectro de sustancias sólidas o líquidas, orgánicas o inorgánicas, dispersas en el aire, procedentes de fuentes naturales y artificiales. La definición de partículas se suele establecer en términos de la vclocidad de depósito de las mismas. En este sentido, se denominan «partículas en suspensión» aquellas con diámetros desde muy por debajo de $0,1 \mu \mathrm{m}$ hasta $5-10 \mu \mathrm{m}$, que permanecen suspendidas durante horas o días; las partículas más grandes caen rápidamente cerca de la fuente que las produce. Otra forma de distinguirlas se refiere a los diferentes métodos usados en su vigilancia de rutina. Se habla de «humos negros») en el caso de partículas con capacidad de tiznar (refíriéndose sólo a la sustancia oscura característica de la combustión incompleta del carbón y de los hidrocarburos) y «total de partículas en suspensión» para las observaciones que se basan directamente en el peso, pudiéndose filtrar por tamaños (por ejemplo, la medición se referirá a $\mathrm{PM}_{10}$ o $\mathrm{PM}_{2,5}$ si pasan a través de un orificio de entrada calibrado con un rendimiento de separación de $50 \%$ para un diámetro aerodinámico de $10 \mu \mathrm{m}$ o $2,5 \mu \mathrm{m}$ respectivamente).

Los elementos que encontramos en las partículas varían mucho según las fuentes locales, pero, en general, los principales componentes son carbono, material alquitranado (hidrocarburos), material soluble en agua, como el sulfato de amonio, y material insoluble conteniendo pequeñas cantidades de hierro, plomo, manganeso y otros elementos, como por ejemplo, el polen.

La revisión de las guías de calidad del aire de la $\mathrm{OMS}^{5}$, así como la futura normativa europea (tabla 5), establecen límites referidos a $\mathrm{PM}_{10} \circ \mathrm{PM}_{2,5}$, fracción respirable de las partículas. Especial importancia tienen estos últimos, por estar asociados con compuestos más tóxicos ${ }^{6}$.

\section{Dióxido de azufre}

El dióxido de azufre es un gas incoloro que a altas concentraciones puede ser detec- 
tado por su sabor y por su olor cáustico e irritante $^{7}$. Se disuelve con facilidad en el agua para formar ácido sulfuroso $\left(\mathrm{H}_{2} \mathrm{SO}_{3}\right)$, el cual se oxida lentamente y forma ácido sulfúrico $\left(\mathrm{H}_{2} \mathrm{SO}_{4}\right)$ con el oxígeno del aire. $\mathrm{El}$ $\mathrm{SO}_{2}$ también puede formar trióxido de azufre $\left(\mathrm{SO}_{3}\right)$, vapor muy reactivo que se combina rápidamente con vapor de agua para formar un aerosol ultra fino de ácido sulfúrico, de gran importancia desde el punto de vista de efectos en salud. Éste puede ser entonces neutralizado por el amonio, formando primero el ácido fuerte bisulfato de amonio $\left(\mathrm{NH}_{4} \mathrm{HSO}_{4}\right)$ y después la sal casi neutra sulfato de amonio $\left[\left(\mathrm{NH}_{4}\right)_{2} \mathrm{SO}_{4}\right]$, dependiendo las tasas de neutralización de las tasas de emisión terrestres de amonio, altas en ciudades y zonas agrícolas, bajas en bosques? ${ }^{7}$ En la atmósfera urbana está representada una amplia gama de compuestos de azufre, pero desde un punto de vista práctico los más importantes son el dióxido de azufre gaseoso, el ácido sulfúrico y los sulfatos.

Las guías de la OMS de calidad del aire para Europa recomiendan no superar concentraciones medias diarias de $125 \mu \mathrm{g} / \mathrm{m}^{3}$ de $\mathrm{SO}_{2}$, con máximos de 10 minutos de $500 \mu \mathrm{g} / \mathrm{m}^{3}$ y valores medios anuales de $50 \mu \mathrm{g} / \mathrm{m}^{3}$.

\section{Dióxido de nitrógeno}

El nitrógeno es un gas sin olor, color ni sabor, que constituye el $78 \%$ del aire. Aunque en condiciones normales no es perjudicial para la salud, se puede combinar con oxígeno para formar diversos óxidos de nitrógeno. La importancia biológica del óxido nítrico (NO) y del dióxido de nitrógeno $\left(\mathrm{NO}_{2}\right)$ es la mejor estudiada; ambos se consideran contaminantes ambientales, y son los más abundante óxidos de nitrógeno producidos por el hombre en áreas urbanas ${ }^{8}$. Tanto el $\mathrm{NO}$ como el $\mathrm{NO}_{2}$ se producen de forma natural en cantidades muy superiores a las generadas por la actividad humana, pero al estar distribuidos sobre toda la superficie terrestre, las concentraciones atmosféricas resultantes son muy pequeñas.
La química atmosférica de los óxidos de nitrógeno es muy compleja. La mayoría de las combustiones liberan sobre todo óxido nítrico, el cual se convierte fácilmente en dióxido de nitrógeno en la atmósfera. La oxidación del $\mathrm{NO}$ a $\mathrm{NO}_{2}$ por oxidantes atmosféricos como $\mathrm{cl}$ ozono, ocurre rápidamente, siendo ésta una de las principales rutas de producción de $\mathrm{NO}_{2}{ }^{9}$. $\mathrm{El} \mathrm{NO}_{2}$ es también un importante contaminante del aire en ambientes interiores, debido al tabaco y a fuentes de combustión de gas inadecuadas o mal ventiladas.

Las guías de la OMS de calidad de aire para Europa ${ }^{9}$ recomendaban no superar valores horarios de $400 \mu \mathrm{g} / \mathrm{m}^{3}$ y medias diarias de $150 \mu \mathrm{g} / \mathrm{m}^{3}$, si bien estos valores van a ser rebajados a $200 \mu \mathrm{g} / \mathrm{m}^{3}$ y entre 40 y $50 \mu \mathrm{g} / \mathrm{m}^{3}$ respectivamente ${ }^{5}$, criterios recogidos en la propuesta de la nueva Directiva de la Unión Europea $^{10}$ (tabla 5).

\section{Monóxido de carbono}

El monóxido de carbono es un gas incoloro, inodoro e insípido, ligeramente menos denso que el aire. En la naturaleza se genera $\mathrm{CO}$ en la producción y degradación de la clorofila, mientras que su origen antropogénico se sitúa en las combustiones incompletas. Este contaminante se combina con la hemoglobina en los glóbulos rojos de la sangre y forma carboxihemoglobina ( $\mathrm{COHb}$ ), siendo la afinidad de la hemoglobina por el $\mathrm{CO}$ unas 250 veces mayor que por el $\mathrm{O}_{2}$. $\mathrm{La}$ formación de $\mathrm{COHb}$ disminuye la capacidad de la sangre de transportar $\mathrm{O}_{2}$ y además aumenta la afinidad de los lugares de unión del $\mathrm{O}_{2}$ por el mismo, lo que interfiere con su liberación en los tejidos y produce hipoxia y alteraciones del funcionamiento celular (en las neuronas, en las células del corazón y de otros músculos). Esto conlleva efectos psicológicos y cardiovasculares (aterosclerosis, arritmias). La exposición crónica a $\mathrm{CO}$ induce la aparición de fenómenos adaptativos tipo aumento del número de glóbulos 
rojos, del volumen sanguíneo y el tamaño del corazón.

Para el monóxido de carbono, los valores máximos recomendados ${ }^{5.9}$ son concentraciones medias en 8 horas de $10 \mathrm{mg} / \mathrm{m}^{3} \mathrm{y}$ valores máximos horarios de $30 \mathrm{mg} / \mathrm{m}^{3}$.

\section{Ozono}

El ozono es un gas altamente reactivo compuesto por tres átomos de oxígeno. Aparece fundamentalmente en dos áreas de la atmósfera, la estratosfera y la troposfera. La mayor parte del ozono atmosférico está en las capas altas de la atmósfera (estratosfera), a 20-50 kilómetros de la superficie terrestre. Presenta un máximo a 25-30 kilómetros, donde se acumula formando una capa llamada ozonosfera, que actúa como filtro de la radiación ultravioleta procedente del sol ${ }^{11}$. Esta capa actualmente está siendo destruida por las emisiones de clorofluorocarburos ( $y$ en menor grado metano y óxidos de nitrógeno), lo que constituye un problema ambiental de enorme importancia, distinto de los problemas ambientales y de salud asociados a la exposición directa al $\mathrm{O}_{3}$ en capas bajas de la atmósfera (troposfera). La presencia de $\mathrm{O}_{3}$ en la troposfera es indeseable, ya que se considera un contaminante con capacidad irritante sobre los tejidos vivos y sobre muchos materiales. Es a este $\mathrm{O}_{3}$ troposférico al que nos referiremos a partir de ahora.

$\mathrm{El}_{3}$ es un contaminante secundario formado en la atmósfera a través de una compleja serie de reacciones químicas de los contaminantes primarios o precursores (óxidos de nitrógeno y compuestos orgánicos volátiles $-\mathrm{COV}-$, fundamentalmente hidrocarburos no metánicos) en presencia de oxígeno atmosférico y luz solar. Sin embargo, estos elementos por sí solos no son suficientes para producir niveles elevados de ozono, los cuales aparecen generalmente cuando las condiciones meteorológicas son adecuadas (temperaturas que exceden los $27-32^{\circ} \mathrm{C}$, mucha luz y ausencia de viento).
En la troposfera no contaminada, los COV de origen natural interaccionan con óxidos de nitrógeno $\left(\mathrm{NO}_{\mathrm{X}}\right)$ y $\mathrm{O}_{2}$ para formar $\mathrm{O}_{3}$, aunque a bajos niveles, aún cuando las condiciones ambientales no son favorables (bajas temperaturas, poca luz solar y mucho viento), como ocurre típicamente en invierno. En los meses de verano, cuando las condiciones de formación de $\mathrm{O}_{3}$ son favorables, sus niveles aumentan en áreas rurales aisladas. Las reacciones químicas esquematizadas son las siguientes ${ }^{11}$ :

$$
\begin{array}{lll}
\mathrm{NO}_{2} & \Leftrightarrow & \mathrm{NO}+\mathrm{O} \\
\mathrm{O}+\mathrm{O}_{2} & \Leftrightarrow & \mathrm{O}_{3} \\
\mathrm{NO}+\mathrm{O}_{3} & \Leftrightarrow & \mathrm{NO}_{2}+\mathrm{O}_{2}
\end{array}
$$

Existe un equilibrio en una serie de reacciones cíclicas entre el $\mathrm{O}_{3}, \mathrm{O}_{2}, \mathrm{O}, \mathrm{NO}_{2}$ y NO , que explica que las medidas de $\mathrm{O}_{3}$ sean menores en el centro de las ciudades (donde hay importantes emisiones de NO de automóviles, fuentes industriales, etcétera) que en áreas rurales. Además, las reacciones que producen $\mathrm{NO}_{2}$ sin destruir $\mathrm{O}_{3}$ alteran el equilibrio, provocando un aumento del $\mathrm{O}_{3}$. La oxidación de COV reactivos fotoquímicamente produce un aumento de radicales libres, capaces de convertir $\mathrm{NO}$ en $\mathrm{NO}_{2}$ sin destruir $\mathrm{O}_{3}$.

Las recomendaciones de las guías de la OMS de calidad de aire ${ }^{5}$ sitúan los valores medios para 8 horas en $120 \mu \mathrm{g} / \mathrm{m}^{3}$.

\section{LA VIGILANCIA DE LA CONTAMINACIÓN ATMOSFÉRICA}

Está generalmente aceptado que niveles altos de contaminación atmosférica se asocian con un exceso de morbi-mortalidad. En este contexto, vienen a la memoria sucesos como el del Valle del Mosa (Bélgica), Donora (Pensilvania) o Londres, que cursaron con la muerte de un número importante de personas expuestas a unos niveles de contaminación extremadamente altos ${ }^{12,13}$. Estos sucesos plantearon la necesidad de limitar la emisión de contaminantes a la atmósfera y, 
sobre todo, de acometer actuaciones de vigilancia de la calidad del aire ambiente ${ }^{14}$.

En España, actualmente, existe un conjunto de redes de vigilancia de la contaminación atmosférica, dependientes de Comunidades Autónomas y Ayuntamientos, que miden valores de inmisión de los principales contaminantes en áreas urbanas y zonas industriales. Los datos registrados en estas redes son suministrados a la Dirección General de Calidad y Evaluación Ambiental del Ministerio de Medio Ambiente que, a su vez, los remite a la Unión Europea. Durante el año 1996 se remitieron a dicha Dirección General datos procedentes de 458 estaciones con sensores manuales y 313 estaciones con sensores automáticos ${ }^{15,16}$. El número de estaciones se incrementa cada año, así como la cantidad de contaminantes analizados. Además de este conjunto de redes que vigilan los niveles de contaminación en zonas densamente pobladas, existe la Red Española de Vigilancia de la Contaminación Atmosférica de Fondo (red EMEP-BAPMoN-CAMP), formada por seis estaciones que miden la concentración de contaminantes en la atmósfera de zonas alejadas de las fuentes de contaminación y que, en breve, se ampliará a 10 estaciones. [La red está integrada en el programa EMEP (European Monitoring Evaluation Programme) nacido del Convenio de Ginebra sobre la contaminación atmosférica transfronteriza a gran distancia, en la red BAPMoN (Background Air Pollution Monitoring Network) de la Organización Meteorológica Mundial y en el Programa CAMP (Comprehensive Atmospheric Monitoring Programme) del Convenio de Oslo y Paris)].

\section{Métodos de captación y análisis de los contaminantes atmosféricos}

La monitorización del aire se refiere a los procedimientos de muestreo y análisis de contaminantes atmosféricos. Para poder medir los niveles de los contaminantes es necesario contar con métodos que produzcan resultados comparables. Para ello, nuestro país tiene definidas las técnicas de referen- cia y los métodos alternativos para la medición de los contaminantes para los que existen valores de referencia.

Los captadores manuales aspiran a lo largo del día una muestra que posteriormente se analiza en laboratorio. Por su parte, los captadores automáticos aspiran una muestra de aire en intervalos de tiempo más cortos, generalmente cada tres minutos, que se analizan en la propia estación. Los datos de estos analizadores se remiten cada media hora a la estación central de la red, donde son almacenados los 48 valores semihorarios diarios. La normativa ${ }^{17}$ que regula los métodos analíticos de gases y partículas recogidos con los captadores manuales de bajo o alto volumen sigue vigente actualmente aunque, poco a poco, dichos captadores se ven desplazados por las Redes Automáticas dotadas de Estaciones de Control de la Contaminación. Esta normativa ya preveía la aparición de métodos alternativos y los valida «siempre que, hechas las oportunas correcciones, sea posible obtener mediciones comprendidas en el intervalo del 10 por 100 , con respecto a la técnica patrón»?.

La contaminación del aire por partículas se ha medido tradicionalmente por la concentración de humos negros. La toma de muestras para humos negros suele hacerse de forma manual, y el método analítico calcula la concentración de humos en la atmósfera, basándose en el ennegrecimiento de un papel de filtro cuando la muestra de aire pasa a través de él ${ }^{9}$. El ennegrecimiento del papel de filtro se mide posteriormente por reflectometría. Este método capta principalmente partículas respirables (típicamente con diámetro de $4,5 \mu \mathrm{m}$ y, en general, menores de $10 \mu \mathrm{m}$ ). Las partículas totales en suspensión se muestrean tanto en redes manuales como automáticas. El método usado por las redes manuales es la gravimetría, basada en el peso del total de partículas en suspensión. El método analítico de las redes automáticas suele ser la atenuación a la radiación beta: las partículas en suspensión de la muestra son retenidas en un papel de fibra 
de vidrio, donde se mide su concentración a partir de la atenuación sufrida por la radiación emitida por una fuente radiactiva de gran estabilidad al atravesar el filtro de fibra de vidrio ${ }^{16}$. En la actualidad comienza a generalizarse el uso de otro método automático basado en la técnica de la balanza inercial.

El método utilizado para las muestras de $\mathrm{SO}_{2}$ por las estaciones manuales es la espectrofotometría con el método de Thorina o el de la pararosanilina ${ }^{9}$, mientras que las estaciones automáticas usan la fluorescencia ultravioleta $^{16}$. La técnica de medida de los óxidos de nitrógeno es la quimiluminiscencia, de referencia para la vigilancia de este contaminante recogida en la Directiva de la Comunidad Europea. El principio de medida se basa en la reacción del $\mathrm{NO}$ con el $\mathrm{O}_{3}$ para formar $\mathrm{NO}_{2}{ }^{16}$. En el caso del $\mathrm{CO}$, la determinación se hace por absorción infrarroja. Se detecta la energía de absorción característica de la molécula de CO. Las líneas del espectro permiten distinguir al $\mathrm{CO}$ de otras sustancias interferentes (dióxido de carbono, vapor de agua, dióxido de azufre y dióxido de nitrógeno ${ }^{16}$. Por último, el método usado en la medición de ozono es la absorción de la radiación ultravioleta, basado en los cambios de intensidad de un haz de luz ultravioleta al atravesar un cierto volumen de gas que contiene moléculas de $\mathrm{O}_{3}{ }^{16}$.

\section{Marco legislativo}

En España, la vigilancia de la contaminación atmosférica se inicia a comienzos de los años 60, labor que fue encomendada a la Escuela Nacional de Sanidad y, más en concreto, al Departamento de Sanidad Ambiental $^{14}$. Progresivamente se fueron incorporando a la vigilancia de la calidad del aire las ciudades con mayores problemas como Madrid, Barcelona, Cartagena, Huelva, Bilbao, Avilés o Puertollano. El marco legislativo de referencia que se maneja en esta época es el Reglamento de $\Lambda$ ctividades Molestas, Insalubres, Nocivas y Peligrosas (RA-
MINP), que en su Anexo 2 establece las concentraciones máximas permitidas en el ambiente interior de las explotaciones industriales, careciéndose de referencias legislativas para el ambiente exterior ${ }^{18}$.

En 1972 se promulga la Ley $38 / 1972$ de 22 de diciembre, sobre protección del ambiente atmosférico ${ }^{1}$, impregnada de los buenos propósitos definidos en las conclusiones de la Primera Conferencia Mundial de Medio Ambiente celebrada en Estocolmo, en la que nació el Programa de Naciones Unidas para el Medio Ambiente (PNUMA) ${ }^{19}$. La Ley es desarrollada por el Decreto 833/ 1975, que derogó otras normativas sectoriales existentes ${ }^{20}$.

Posteriormente se publican la Orden de 10 de agosto de 1976, por la que se establecen las normas técnicas para el análisis y valoración de contaminantes de naturaleza química $^{17}$, y la Orden 18 de octubre de 1976, de prevención y corrección de la contaminación atmosférica industrial ${ }^{21}$.

La incorporación de España a la Comunidad Económica Europea llevó aparejada la adecuación de nuestros criterios de calidad del aire exterior a las pautas europeas. Esto hizo que los niveles de $\mathrm{SO}_{2}$ y partículas en suspensión se viesen modificados en varias ocasiones, a través del Real Decreto 1613/ $1985^{22}$ y el Real Decreto 1321/1992 ${ }^{23}$. Los niveles de $\mathrm{NO}_{2}$ y plomo, por el Real Decreto $717 / 1987^{24}$ y más recientemente se ha publicado Real Decreto $1494 / 1995^{25}$ sobre contaminación atmosférica por ozono. En la tabla 4 se resumen los niveles establecidos por la normativa actualmente vigente.

Paralelamente al establecimiento de criterios de calidad del aire se han arbitrado medidas correctoras para reducir la emisión de contaminantes. Por ello, existe también un marco legislativo que limita las emisiones de vehículos automóviles, define las características, calidades y condiciones de empleo de carburantes y combustibles, limita las emisiones a la atmósfera de determina- 
dos agentes contaminantes procedentes de grandes instalaciones de combustión y de las de incineración de residuos municipales.

Actualmente, como consecuencia de la aplicación del V Programa de Medio Ambiente elaborado por la Comisión de las Comunidades Europeas que lleva por título «Hacia un Desarrollo Sostenible: Programa comunitario de política y Actuación en materia de Medio Ambiente y Desarrollo Sostenible ${ }^{26}$, se han publicado diferentes Directivas que pretenden avanzar en la prevención de la contaminación y, por ende, en la prevención de los efectos deletéreos derivados de la misma. Así, la Directiva 96/62/CE del Consejo ${ }^{27}$, sobre evaluación y gestión de la calidad del aire ambiente, conocida como la Directiva sobre calidad del aire y la Decisión del Consejo de 27 de enero de $1997^{28}$, por la que se establece el intercambio recíproco de información y datos de las redes y estaciones aisladas de medición de la contaminación atmosférica de los Estados Miembros han sido promulgadas con el «fin de definir y establecer objetivos de calidad del aire ambiente al objeto de evitar, prevenir o reducir los efectos nocivos para la salud humana y para el medio ambiente en su conjunto» En desarrollo de la Directiva 96/62/CE se está tramitando actualmente una propuesta de Directiva relativa a los valores límite de dióxido de azufre, óxidos de nitrógeno, partículas y plomo en el aire ambiente que se recogen en la tabla $5^{10}$.

\section{EL PAPEL DE LA SALUD PÚBLICA}

Para la Salud Pública, en concreto para la Sanidad Ambiental, la contaminación atmosférica representa una necesidad constante de acometer acciones en un doble sentido. Por un lado, es necesario plantear nucvas líncas de investigación de contaminantes de mayor abundancia en España y en el área mediterránea, así como continuar con las líneas de investigación abiertas en orden a mejorar las mediciones de exposición y las de efecto de los contaminantes atmosféricos sobre la sa- lud. La calidad en la evaluación de la exposición, el principal problema de los estudios de epidemiología ambiental ${ }^{29}$, es un elemento imprescindible para poder estudiar los efectos de los contaminantes en la salud. Cuando los niveles de contaminación son altos, la epidemiología ha conseguido demostrar sus efectos en la salud. Sin embargo, cuando los niveles de exposición son bajos, aparecerían dificultades en la detección de dichos efectos.

A pesar de las dificultades que presentan los estudios de contaminación atmosférica, en años recientes numerosos estudios han puesto de manifiesto la posible relación entre niveles bajos de contaminación atmosférica y la morbi-mortalidad de las poblaciones expuestas ${ }^{30-37}$. Entre ellos, destaca una iniciativa europea, el proyecto APHEA (short term effects of Air Pollution on Health: an European Approach) ${ }^{38}$, que ha promovido el estudio de los efectos a corto plazo de la contaminación atmosférica en la salud mediante un estudio multicéntrico en el que han participado 11 grupos europeos y 15 ciudades (siendo Barcelona la única ciudad española). Siguiendo la metodología desarrollada y estandarizada en este proyecto, en España se puso en marcha el proyecto EMECAM. Como continuación del proyecto $A P H E A$, ya ha comenzado el $A P H E A I I$ (short term effects of air pollution on health: a European approach to methodology, dose-response assessment and evaluation of public health significance $)^{39}$.

Por otro lado, la Salud Pública ha de establecer líneas de intervención que traduzcan los resultados de estas investigaciones en recomendaciones dirigidas a las políticas ambientales sectoriales, fundamentalmente en el campo de la vigilancia de la calidad del aire, que en España es competencia de las autoridades de medio ambiente (tanto a escala estatal, como autonómica y local). Las recomendaciones deberían referirse fundamentalmente a la revisión de los niveles establecidos en la legislación. También, siguiendo iniciativas desarrolladas por otros 
países, los profesionales de la Salud Pública tienen la posibilidad de dirigir sus esfuerzos a la creación de sistemas integrados de vigilancia activa, que registren en tiempo cuasi real, la evolución de los niveles de contaminantes en la atmósfera (no sólo químicos, también bióticos como polen o esporas), y las variables meteorológicas que interactúan con la exposición a esos contaminantes, así como las variables que miden los efectos en términos de morbimortalidad. Un sistema así configurado, capaz de generar predicciones de la evolución temporal y espacial de los factores de riesgo y de la consecuente evolución espacial y temporal de los indicadores de salud, podría retroalimentar al propio sistema sanitario, no sólo en el campo de la investigación sino en el de la propia asignación y gestión de recursos. Y, lo que es más importante, permitiría disponer de una información de alto valor desde el punto de vista de la prevención, que es, en definitiva, el objetivo principal de la salud pública.

\section{BIBLIOGRAFÍA}

1. Boletín Oficial del Estado. Ley $38 / 1972$, de 22 de diciembre de Protección del Medio Ambiente Atmosférico. BOE 26/12/72.

2. Elsom D. La contaminación atmosférica. Madrid: Cátedra; 1990.

3. Graedel TE, Grutzen PJ. Una atmósfera cambiante. Inves Ciencia 1989;158:22-32.

4. Sanz J. La contaminación atmosférica. Bilbao: Ministerio de Obras Públicas y Transporte; 1991.

5. World Health Organization. Update and revision of the air quality guidelines for Europe. Offset. Copenhagen: World Health Organization; 1995.

6. Spurny, KR. Atmospheric particulate pollutants and environmental health. Arch Environ Health 1996;5:415-416.

7. World Health Organization. Sulphur oxides and suspended particulate matter. Environ Health Criteria 8. Geneve: World Health Organization; 1979.

8. World Healih Organization. Oxides of Nitrogen. Environ Health Criteria 4. Geneve: World Health Organization; 1977.
9. World Health Organization. Air Quality Guidelines for Europe. WHO Regional Publications, European Series No 23. Copenhagen, 1987.

10. Comisión de las Comunidades Europeas. Propuesta de Directiva del Consejo relativa a los valores límite de dióxido de azufre, óxidos de nitrógeno, partículas y plomo en el aire ambiente. Bruselas: Comisión de las Comunidades Europeas; 1997.

11. Mckee DJ. Tropospheric ozone: human health and agricultural impacts. Boca Raton. Lewis publishers; 1993.

12. Moeller DW. Environ Health . London: Harvard University Press; 1992.

13. Abercrombie GF. December fog in London and the emergency bed service. Lancet 1953.

14. Ministerio de Sanidad y Consumo. Red nacional de vigilancia y prevención de la contaminación atmosférica. Madrid: Ministerio de Sanidad y Consumo; 1988.

15. Ministerio de Medio Ambiente. Medio ambiente en España 1996. Madrid: Ministerio de Medio Ambiente;1997.

16. Ministerio de Obras Públicas, Transportes y Medio Ambiente. Estaciones y redes de vigilancia de la contaminación atmosférica en España. Descripción técnica y gráfica. Serie Monografias. Madrid: Ministerio de Obras Públicas, Transportes y Medio Ambiente; 1993.

17. Boletín Oficial del Estado. Orden de 10 de agosto de 1976, por la que se establecen las normas técnicas para el análisis y valoración de contaminantes de naturaleza química. BOE 5/11/1976.

18. Boletín Oficial del Estado. Decreto 2414/1961, de 30 de noviembre, por el que se aprueba el Reglamento de actividades molestas, insalubres, nocivas y peligrosas. BOE 7/12/1961.

19. Organización de Naciones Unidas. Declaración de NU sobre el medio ambiente: proclamaciones y principios. Conferencia de Estocolmo. Estocolmo: Organización de Naciones Unidas; 1972.

20. Boletín Oficial del Estado. Decreto 833/1975 de 6 de febrero, por el que se aprueba el Reglamento de la Ley 38/72. BOE 22/4/1975.

21. Boletín Oficial del Estado. Orden de 18/10/1976 de prevención y corrección de la contaminación atmosférica industrial. BOE 3/12/1976.

22. Boletin Oficial del Estado. Real Decreto 1613/85 de 1 de agosto, por el que se establecen nuevas normas de calidad del aire en lo referente a contaminación por dióxido de azufre y partículas en suspensión. BOE 12/9/1985. 
23. Boletín Oficial del Estado. Real Decreto 1321/92 de 30 de octubre, por el que se modifica el RD $1613 / 85$ y se establecen nuevas normas de calidad del aire en lo referente a contaminación por dióxido de azufre y partículas en suspensión. BOE 2/12/1992.

24. Boletin Oficial del Estado. Real Decreto 717/87 de 27 de mayo, por el que se establecen nuevas normas de calidad del aire en lo referente a contaminación por dióxido de nitrógeno y plomo. $\mathrm{BOE}$ 6/6/1987.

25. Boletín Oficial del Estado. Real Decreto 1494/95 de 8 de septiembre, sobre contaminación atmosférica por ozono. BOE 26/9/1995.

26. Comisión de las Comunidades Europeas. Hacia un desarrollo sostenible: programa comunitario de política y actuación en materia de medio ambiente y desarrollo sostenible. Bruselas: Comisión de las Comunidades Europeas; 1992.

27. Diario Oficial de las Comunidades Europeas. Directiva 96/62/CE de 27 de septiembre, sobre evaluación y gestión del aire ambiente. DOCE 21/11/1996.

28. Diario Oficial de las Comunidades Europeas. Decisión del Consejo de 27 de enero de 1997, por la que se establece un intercambio recíproco de información y datos de las redes y estaciones aisladas de medición de la contaminación atmosférica en los Estados miembros. DOCE 5/2/1997.

29. Sunyer J. Característiques i funcions de l'epidemiologia ambiental. Gac Sanit 1990;19: 145-156.

30. Ballester Díez F, Merino Egea C, Pérez-Hoyos S. La asociación entre contaminación atmosférica y mortalidad: una revisión de los estudios epidemiológicos recientes. Rev Esp Salud Pública 1995;69:177-188.

31. Spix C, Anderson HR, Schwartz J, Vigotti MA, LeTertre A, Vonk JM et al. Short-term effects of air pollution on hospital admissions of respiratory diseases in Europe: a quantitative summary of APHEA study results. Arch Fnviron Health 1998;53:54-64.

32. Sunyer J, Antó JM, Murillo C, Saez M. Effects of urban air pollution on emergency room admissions for chronic obstructive pulmonary disease. Am J Epidcmiol 1991;134:227-286.

33. Schwartz J, Marcus A. Mortality and air pollution in London: a time series analysis. Am J Epidemiol 1990;131:185-94.

34. Seaton A, MacNee W, Donaldson K, Godden D. Particulate air pollution and acute health effects. Lancet 1995;345:176-78.

35. Toulomi G, Pocock SJ, Katsouyanni K, Trichopoulos $D$. Short-term effects of air pollution on daily mortality in Athens: a time-series analysis. Int J Epidemiol 1994;23:957-967.

36. Guillén JJ et al. Afluencia inusual por asma y enfermedad pulmonar obstructiva crónica en urgencias hospitalarias y contaminación por $\mathrm{SO}_{2}$ en Cartagena. Rev Esp Salud Pública 1995;69:305-314.

37. Diaz J, Alberdi JC, Montero JC, Mirón IJ. Asociación entre la contaminación atmosférica por dióxido de azufre y partículas totales en suspensión y la mortalidad diaria en la ciudad de Madrid (1986-1992). Gac Sanit 1998; 12:207-215.

38. Katsouyanni K, Schwartz J, Spix C, Touloumi G, Zmirou D, Zanobetti A y cols. Short term effects of air pollution on health: a European approach using epidemiologic time series data: the APHEA protocol. J Epidemiol Community Health 1996; 50(Suppl 1):S12 S18.

39. Katsouyanni K, Toulomi G, Samoli E, Wichmann E, Spix C, Anderson HR et al. Short-term effects of air pollution on health: a European approach to methodology, dose-response assessment and evaluation of public health significance (APHEA 2 project). Proceedings of an EC Research Workshop; 1998 March 22-25; Athens. Luxembourg: Office for Official Publications of the European Communities, 1998. 


\section{FE DE ERRATAS}

En el trabajo Contaminantes atmosféricos y su vigilancia Emiliano Aránguez, José María Ordóñez, Javier Serrano, Nuria Aragonés, Rosalia Fernández-Patier, Ana Gandarillas e
Iñaki Galán: 73 (2): 123-132, se produjo un error consistente en no publicar las tablas a las que los autores se refieren en el texto. A continuación se publican las mismas.

Tabla 1

Contaminantes atmosféricos: fuentes antropogénicas y concentraciones de fondo

\begin{tabular}{|c|c|c|c|c|c|}
\hline \multirow{3}{*}{ Contaminante } & \multicolumn{2}{|c|}{ Fuente antropogénica } & \multicolumn{3}{|c|}{ Concentración de fondo $*$} \\
\hline & \multirow{2}{*}{ Procesos } & \multirow{2}{*}{ Actividades } & \multicolumn{2}{|c|}{ Europa ** } & \multirow{2}{*}{$\frac{\text { España }}{\text { Rural }}$} \\
\hline & & & Urbana & Rural & \\
\hline Humos negros & \multirow{2}{*}{$\begin{array}{l}\text { Combustión de carbón y } \\
\text { petróleo, combustiones in- } \\
\text { completas }\end{array}$} & \multirow{2}{*}{$\begin{array}{l}\text { Calefacciones, centrales } \\
\text { térmicas, tráfico, incinera- } \\
\text { ción de residuos }\end{array}$} & $\begin{array}{c}10-40^{1} \\
100-250^{2}\end{array}$ & $0-10^{1}$ & \\
\hline PST & & & $\begin{array}{c}50-150^{1} \\
200-400^{2} \\
\end{array}$ & & $20-46^{1}$ \\
\hline $\mathrm{SO}_{2}$ & $\begin{array}{l}\text { Combustión de materiales } \\
\text { que contienen azufre, acti- } \\
\text { vidades industriales }\end{array}$ & $\begin{array}{l}\text { Calefacciones, tráfico, in- } \\
\text { dustrias }\end{array}$ & $<100^{1}$ & $5-25^{1}$ & $2-3^{1}$ \\
\hline $\mathrm{NO}_{2}$ & $\begin{array}{l}\text { Proccsos de combustión } \\
\text { (oxidación del N atmosféri- } \\
\text { co), fabricación de ácido ní- } \\
\text { trico, explosivos }\end{array}$ & $\begin{array}{l}\text { Tráfico, industrias, calefac- } \\
\text { ciones, interiores mal venti- } \\
\text { lados }\end{array}$ & $\begin{array}{c}20-90^{1} \\
130-400^{2}\end{array}$ & $0.4-9.4^{1}$ & $3-4^{1}$ \\
\hline $\mathrm{CO}$ & $\begin{array}{l}\text { Combustiones incompletas, } \\
\text { disociación de } \mathrm{CO}_{2} \text { a altas } \\
\text { temperaturas, producción } \\
\text { de } \mathrm{CO} \text { a partir de } \mathrm{CO}_{2}\end{array}$ & $\begin{array}{l}\text { Tráfico, calefacciones, in- } \\
\text { dustrias, interiores mal ven- } \\
\text { tilados }\end{array}$ & $<20^{3}$ & $0.01-0.23$ & \\
\hline $\mathrm{O}_{3}$ & $\mathrm{NO}_{x}$ y COV + luz solar & $\begin{array}{l}\text { Las que generan } \mathrm{NO}_{\mathrm{x}} \mathrm{y} \\
\mathrm{COV}\end{array}$ & & $40-60^{4}$ & \\
\hline
\end{tabular}

${ }^{1}$ Media anuai; ${ }^{2}$ Máxima media diaria; ${ }^{3}$ Media 8 horas; ${ }^{4}$ Percentil $50-24$ horas.

* Concentraciones expresadas en $\mu \mathrm{g} / \mathrm{m}^{3}$, excepto para el $\mathrm{CO}\left(\mathrm{mg} / \mathrm{m}^{3}\right)$.

Fuentes: ** WHO. Air Quality Guidelines for Europe. WHO Regional Publications, European Series No 23. Copenhagen, 1987.

*** Red EMEP/BAPMON/CAMP. Ministerio de Medio Ambiente. Medio ambiente en España 1996. Madrid, 1997.

Tabla 2

Factores de conversión entre unidades de medida de la concentración de contaminantes en el aire

\begin{tabular}{|ccccc|}
\hline & $\mathrm{SO}_{2}$ & $\mathrm{NO}_{2}$ & $\mathrm{CO}$ & $O_{3}$ \\
\cline { 2 - 5 } $1 \mathrm{ppm}$ & $2860 \mu \mathrm{g} / \mathrm{m}^{3}$ & $1880 \mu \mathrm{g} / \mathrm{m}^{3}$ & $1,145 \mathrm{mg} / \mathrm{m}^{3}$ & $2000 \mu \mathrm{g} / \mathrm{m}^{3}$ \\
\hline
\end{tabular}

Fuente: WHO Air Quality Guidelines for Europe. WHO Regional Publications, European Series No 23. Copenhagen, 1987. 
Tabla 3

Principales métodos analíticos para medir niveles de inmisión de contaminantes atmosféricos

\begin{tabular}{|lcc|}
\hline Contaminante & Muestreo manual & Muestreo autómatico \\
\hline Humos negros & Reflectometría & \\
Partículas & Gravimetría (Alto volumen) & Atenuación radiación beta \\
& Thorina (Técnica Patrón) & TEOM (balanza inercial) \\
$\mathrm{SO}_{2}$ & Pararosanilina & Fluorescencia UV \\
& - & Quimiluminiscencia \\
$\mathrm{NO}_{2}$ & - & Absorción infrarrojo no dispersivo \\
$\mathrm{CO}$ & - & Absorción UV \\
$\mathrm{O}_{3}$ & - &
\end{tabular}

Fuente: elaboración propia.

Tabla 4

Valores límite de inmisión establecidos en la legislación vigente

\begin{tabular}{|c|c|c|c|c|}
\hline Contaminante & $\begin{array}{l}\text { Periodo de } \\
\text { referencia }\end{array}$ & Valor limite & Indicador considerado & Ohservaciones \\
\hline \multirow[t]{2}{*}{$\begin{array}{l}\text { Particulas (PST) } \\
\text { R.D. } 1321 / 1992\end{array}$} & $\begin{array}{l}\text { Anual (1 abril a } 31 \text { de } \\
\text { marzo) }\end{array}$ & $150 \mu \mathrm{g} / \mathrm{m}^{3}$ & Valores medios diarios & \\
\hline & & $300 \mu \mathrm{g} / \mathrm{m}^{3}$ & $\begin{array}{l}\text { Percentil } 95 \text { de las me- } \\
\text { dias diarias }\end{array}$ & \\
\hline \multirow{3}{*}{$\begin{array}{l}\text { Partículas (humos } \\
\text { negros }-\mathrm{HN}-\text { ) } \\
\text { R.D. } 1321 / 1992\end{array}$} & $\begin{array}{l}\text { Anual ( } 1 \text { abril a } 31 \text { de } \\
\text { marzo) }\end{array}$ & $80 \mu \mathrm{g} / \mathrm{m}^{3}$ & $\begin{array}{l}\text { Mediana de los prome- } \\
\text { dios diarios }\end{array}$ & \\
\hline & & $250 \mu \mathrm{g} / \mathrm{m}^{3} *$ & $\begin{array}{l}\text { Percentil } 98 \text { de las me- } \\
\text { dias diarias }\end{array}$ & $\begin{array}{l}\text { * No se deben sobre- } \\
\text { pasar más de } 3 \text { días } \\
\text { consecutivos }\end{array}$ \\
\hline & $\begin{array}{l}\text { Período invernal ( } 1 \\
\text { octubre a } 31 \text { marzo) }\end{array}$ & $130 \mu \mathrm{g} / \mathrm{m}^{3}$ & $\begin{array}{l}\text { Mediana de las medias } \\
\text { diarias }\end{array}$ & \\
\hline \multirow[t]{6}{*}{$\begin{array}{l}\text { Dióxido de azufre } \\
\text { R.D. } 1321 / 1992\end{array}$} & $\begin{array}{l}\text { Anual (1 abril a } 31 \text { de } \\
\text { marzo) }\end{array}$ & $\begin{array}{l}80 \mu \mathrm{g} / \mathrm{m}^{3} \\
(\mathrm{si} \quad \mathrm{HN}>40 \text { o } \mathrm{PST}>150)\end{array}$ & Mediana de los valores & \multirow{6}{*}{$\begin{array}{l}\text { * No se deben sobre- } \\
\text { pasar durante más de } \\
\text { tres dias consecutivos }\end{array}$} \\
\hline & & $\begin{array}{l}120 \mu \mathrm{g} / \mathrm{m}^{3} \\
(\text { si } \mathrm{HN} \leq 40 \text { o PST } \leq 150)\end{array}$ & medios diarios & \\
\hline & & $\begin{array}{l}250 \mu \mathrm{g} / \mathrm{m}^{3} * \\
(\mathrm{si} \mathrm{HN}>150 \circ \text { PST }>350)\end{array}$ & $\begin{array}{l}\text { Percentil } 98 \text { de todos los } \\
\text { valores medios diarios }\end{array}$ & \\
\hline & & $\begin{array}{l}350 \mu \mathrm{g} / \mathrm{m}^{3 *} \\
\text { (si IIN } \leq 150 \text { o } \text { PST } \leq 350 \text { ) }\end{array}$ & & \\
\hline & Período invernal & $\begin{array}{l}130 \mu \mathrm{g} / \mathrm{m}^{3} \\
(\text { si } \mathrm{HN}>60 \text { o } P S T>200)\end{array}$ & \multirow{2}{*}{$\begin{array}{l}\text { Mediana de los valores } \\
\text { medios diarios }\end{array}$} & \\
\hline & & $\begin{array}{l}180 \mu \mathrm{g} / \mathrm{m}^{3} \\
\text { (si HN } \leq 60 \text { o PST } \leq 200 \text { ) }\end{array}$ & & \\
\hline $\begin{array}{l}\text { Dióxido de nitrógeno } \\
\text { R.D. } 717 / 1987\end{array}$ & $\begin{array}{l}\text { Anual ( } 1 \text { enero a } 31 \\
\text { diciembre) }\end{array}$ & $200 \mu \mathrm{g} / \mathrm{m}^{3}$ & $\begin{array}{l}\text { Percentil } 98 \text { de las me- } \\
\text { dias horarias o periodos } \\
\text { inferiores a la hora }\end{array}$ & $\begin{array}{l}\text { Dato válido si missings } \\
<25 \%\end{array}$ \\
\hline \multirow{5}{*}{$\begin{array}{l}\text { Monóxido de carbono } \\
\text { D. } 833 / 1975\end{array}$} & Media hora & $45 \mathrm{mg} / \mathrm{m}^{3}$ & Concentración máxima & \\
\hline & Ocho horas & $15 \mathrm{mg} / \mathrm{m}^{3}$ & Media & \multirow{4}{*}{$\begin{array}{l}\text { Emergencia de }(*) \text { pri- } \\
\text { mer, }\left(^{* *}\right) 2^{\circ} \text { grado y } \\
(* * *) \text { total }\end{array}$} \\
\hline & Día & $34 \mathrm{mg} / \mathrm{m}^{3}\left(^{*}\right)$ & Media & \\
\hline & & $46 m g / m^{3}(* *)$ & & \\
\hline & & $60 \mathrm{mg} / \mathrm{m}^{3}(* * *)$ & & \\
\hline
\end{tabular}




\begin{tabular}{|lllll|}
\hline Contaminante & $\begin{array}{c}\text { Periodo de } \\
\text { referencia }\end{array}$ & Valor limite & Indicador considerado & Observaciones \\
\hline $\begin{array}{l}\text { Ozono } \\
R . D .1494 / 1995\end{array}$ & Ocho horas & $110 \mu \mathrm{g} / \mathrm{m}^{3}$ & Media & $\begin{array}{l}\text { Umbral de protección } \\
\text { de la salud }\end{array}$ \\
& Una hora & $180 \mu \mathrm{g} / \mathrm{m}^{3}$ & Media & $\begin{array}{l}\text { Umbral de información } \\
\text { a la población } \\
\text { Umbral de alerta a la } \\
\text { población }\end{array}$ \\
\hline
\end{tabular}

Fuente: elaboración propia.

Tabla 5

Propuesta de Directiva relativa a los valores límite de dióxido de azufre, óxidos de nitrógeno, partículas $y$ plomo en el aire ambiente

\begin{tabular}{|c|c|c|c|}
\hline Contaminante & Periodo de referencia & Valor limite & Observaciones \\
\hline \multirow[t]{2}{*}{ Partículas $\left(\mathrm{PM}_{10}\right)^{1}$} & Anual & $30 \mu \mathrm{g} / \mathrm{m}^{3}$ & \\
\hline & 24 horas & $50 \mu \mathrm{g} / \mathrm{m}^{3}$ & Se podrá sobrepasar 25 días al año \\
\hline \multirow[t]{2}{*}{ Partículas $\left(\mathrm{PM}_{10}\right)^{2}$} & Anual & $20 \mu \mathrm{g} / \mathrm{m}^{3}$ & \\
\hline & 24 horas & $50 \mu \mathrm{g} / \mathrm{m}^{3}$ & Se podrá sobrepasar 7 días al año \\
\hline \multirow[t]{2}{*}{ Dióxido de azufre ${ }^{1}$} & 1 hora & $350 \mu \mathrm{g} / \mathrm{m}^{3}$ & Se podrá sobrepasar 24 horas al año. \\
\hline & 24 horas & $125 \mu \mathrm{g} / \mathrm{m}^{3}$ & Se podrá sobrepasar 3 dias al año \\
\hline \multirow[t]{2}{*}{ Dióxido de nitrógeno ${ }^{2}$} & 1 hora & $200 \mu \mathrm{g} / \mathrm{m}^{3}$ & Se podrá sobrepasar 8 horas al año \\
\hline & Anual & $40 \mu \mathrm{g} / \mathrm{m}^{3}$ & \\
\hline Plomo ${ }^{1}$ & Anual & $0.5 \mu \mathrm{g} / \mathrm{m}^{3}$ & \\
\hline
\end{tabular}

Entraria en vigor a partir del 1,1.2005.

2 Entraria en vigor a partir del 1.1.2010.

Fuente: Comisión de las Comunidades Europeas. Propuesta de Directiva del Consejo relativa a los valores límite de dióxido de azufre, óxidos de nitrógeno, particulas y plomo en el aire ambiente. Bruselas, 1997. 